\title{
Acceleration feedback control for enhancing dynamic stiffness of fast tool servo system considering the sensor imperfections
}

\author{
Fei Ding Xichun Luo Yukui Cai Wenlong Chang \\ University of Strathclyde
}

\begin{abstract}
Lorentz type fast tool servo devices have found wide applications in freeform machining but they face problems of insufficient stiffness with large depth of cut. Acceleration feedback control is an alternative way to enhance the dynamic stiffness without the need for a large inertia, which is strictly limited in fast tool servo devices. However, the current knowledge gap in the understanding of the influences of limited sensor bandwidth and sensor noises on positioning performance has impeded the application of acceleration feedback control approach in fast tool servo devices. This paper established an analytical model to reveal, for the first time, how much positioning errors are caused by the added sensor noises and how the acceleration feedback technique changes the closed loop stiffness. The measured positioning error spectrum agrees with the modelled one with different acceleration gains. The stiffness model is verified through frequency response tests. It is found that the dynamic stiffness is significantly improved by 5.6 folds within the acceleration sensor bandwidth, while the stiffness deteriorates at frequencies beyond the bandwidth due to the low-pass characteristics in the acceleration loop. The stiffness analysis results are further verified in the intermittent facing cut experiments. The measured surface form errors can be mapped to the low frequency and high frequency vibrations caused by the cutting forces. The analysis model provides a theoretical basis for adopting acceleration feedback technique, paving the way for its practical implementations in ultraprecision applications.
\end{abstract}


Keywords: Fast tool servo; Diamond turning; Acceleration sensor; Dynamic stiffness; Positioning error;

\section{Introduction}

Recent years have seen an upsurge of the demands for freeform and micro-structured parts in the optical industries. Fast Tool Servo (FTS) devices are designed to manufacture these freeform and micro-structured surfaces [1]. They are also used in the manufacturing of pistons in the automotive industry $[2,3]$. However, the machining efficiency is limited by the maximum depth of cut for each cycle and there is also a trend to apply the FTS techniques to cutting brittle materials [4]. The large cutting forces will induce form errors and therefore pose great challenges for conventional FTS devices [5]. The FTS cutting system needs more dynamic stiffness to overcome the force disturbances. Dynamic stiffness of a cutting system is also closely related to the cutting stability [6].

The dynamic stiffness along the movement direction sources from both passive and active stiffness. The passive stiffness is determined by the physical properties of the moving parts, like spring coefficient, damping and inertia, while the active one comes from the closed loop control action [7]. In general, enhanced dynamic stiffness can be achieved by designing the part with a large inertia. However, this is not applicable for fast tool servo applications because the inertia cannot be increased much due to power limits of the drive. To the best of the authors' knowledge, current researches have been focused on structural design of FTS. Dynamic stiffness of a FTS and how to improve it from the control point of view are rarely studied.

Acceleration feedback is a control technique using an auxiliary accelerometer together with the position sensor to measure the status of the tool and generate control output. It can be used to increase the motion bandwidth of fast positioners [8]. The idea of using acceleration information to enhance system dynamic performance is firstly proposed by Hori [9] and Lorentz [10,11]. When only displacement sensor is used as the feedback source, other information like velocity has to be derived from the encoder by numerical differentiation, with significant noises [12]. Direct measurement of the acceleration signal averts this problem and it can also be used to correct the noise of the velocity signal as estimated from the displacement sensor [13]. 
Wittmann et al. presented a hybrid current and acceleration control approach which is equivalent to a disturbance observer and discussed the noise sensitivity and load sensitivity [14]. Tian et al. extended the acceleration control concept to a magnetic levitation system and showed that the sinusoidal disturbance is attenuated [15]. Different arrangements of the current loop and acceleration loop has been tested and compared with cascaded PI controller by Griese and Maas [16]. Dumanli et al. fused the acceleration and encoder signals by kinematic state observer to estimate the acceleration and jerk and then a full state feedback is realised. They have observed enhanced stiffness at the antiresonant and resonant frequencies but didn't pay attention to the stiffness at other frequency ranges [17]. Katsura et al. proposed the PAIDO idea in [18], where they used the displacement and acceleration signals to compose the disturbance observer in a way similar to a complimentary filter. Although the major purpose of adopting acceleration feedback is to reduce the errors caused by forces, it is also proved to be helpful in suppressing crosstalk from other slides in lithography machines [19].

The above control strategies can be grouped into two types: the feedforward compensation type and acceleration control type. In the first type, the signals are used to estimate the disturbance force through observers and then compensate for it. In the second type, the acceleration is treated as a new process variable to be controlled. These two types have been proved to be mathematically the same by Suzumura [20]. There are also other ways to incorporate acceleration signal in control loops: Acceleration signal is used to actively suppress structural chatter vibration [21,22]. Abir et al. used the acceleration signal to estimate the machine tool frame vibration and tried to compensate for it in the controller [23,24]. Accelerometers are also commonly found in active vibration isolation systems $[25,26]$.

Despite the improved system resistance to disturbances with acceleration feedback, it hasn't been adopted in ultra-precision machining systems. A very importance reason is that practical sensors always have a limited bandwidth and a certain level of electronic noises. Previous studies have been focusing on the design of controller algorithms and the estimation of acceleration information. Few have pointed out how the stiffness changes over the whole frequency range regards to the sensor bandwidth. And the tool positioning error caused by the additional sensor noise is another important 
factor to be considered in ultra-precision positioning application. These topics haven't been discussed in the literature yet.

This paper addresses these issues by establishing a dynamic stiffness model of a fast tool positioning system. The effects of the low-pass characteristics in the acceleration loop are discussed. The error contribution of the acceleration sensor is analysed and experimentally verified. The dynamic stiffness is tested through both frequency response test and intermittent diamond turning. The results are compared with the estimation from the model.

\section{Methodology}

\subsection{Acceleration feedback control algorithm}

The conventional motion control scheme consists of a cascade current loop within the position and velocity loops, as shown in Figure 1 a). The current loop is closed upon a current sensing resistor in series with the motor windings. So the current feedback only depicts how much force is generated by the motor. Other disturbances such as cutting force or cable induced forces cannot be sensed until they induce errors on the velocity signal or position signal. The estimation of disturbance force from velocity signal is a first order differentiation process and this will introduce a half sampling period delay. In most case, the velocity signal is derived from the position sensor by numerical differentiation. This means another delay in the loop [12].

In the acceleration feedback approach, accelerometers are used to measure the momentum stage acceleration. Since the acceleration signal is strictly proportional to the net force experienced by the stage, the forces can be sensed without the differentiation delays. Thus the controller can compensate for the disturbance force in time by generating an opposite motor force before they affect the positioning accuracy, creating a "virtual mass" effect. As shown in Figure $1 \mathrm{~b}$ ), the cascade acceleration loop is closed by feeding back the measured acceleration signal directly to the controller. The output of the position or velocity loop serves as the set point of the acceleration loop. 


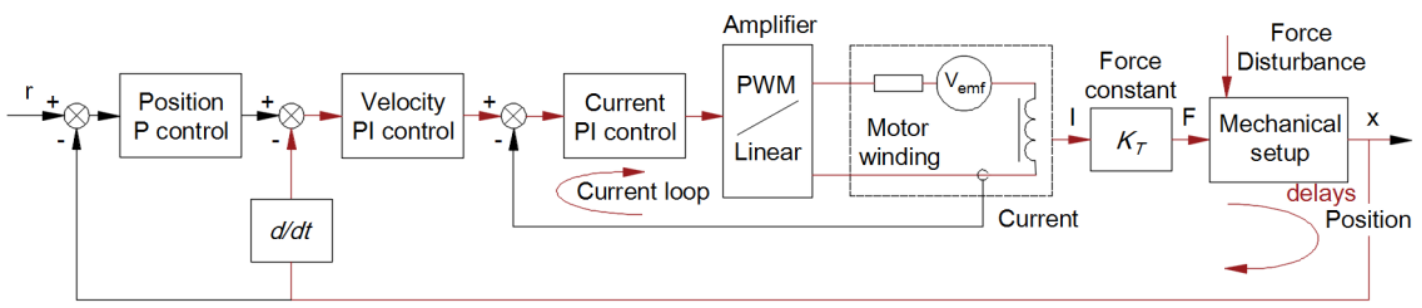

a) Conventional motion control with position feedback

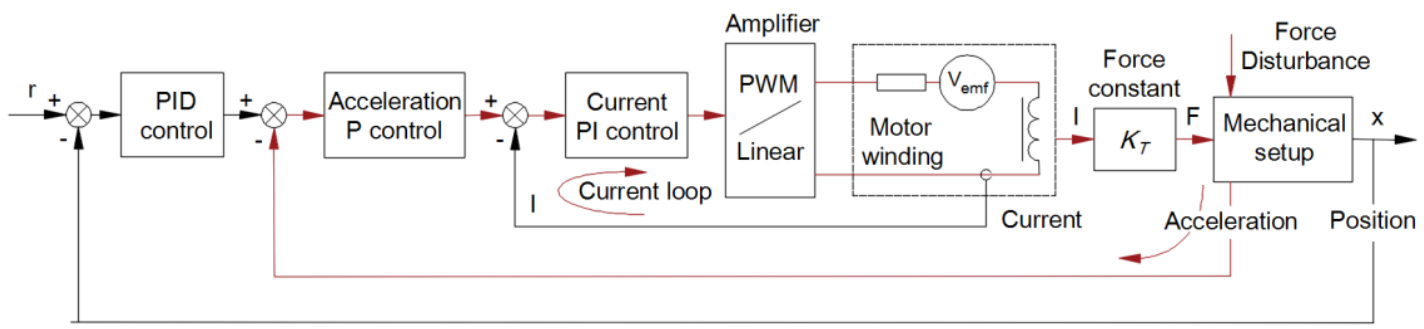

b) Motion control with position and acceleration feedback

Figure 1: Comparison between conventional and acceleration control loops (Red arrows show the shortest path from disturbance to controller)

Figure 2 illustrates an analytical model of the acceleration feedback control system shown in Figure $1 \mathrm{~b}$ ). In this model, the position loop is closed by a typical PID control algorithm with first order low-pass action on the derivative term. The mechanical setup is simplified as a single body mass-spring-damper system with moving mass $M$, support stiffness $K$ and damping coefficient $C$. The acceleration of the moving mass is directly measured by an accelerometer. The frequency responses of the accelerometer and the capacitive displacement sensor are denoted by $H_{\text {acc }}$ and $H_{\text {capa }}$ respectively. $H_{I}$ represents the transfer function of the closed current loop and $K_{T}$ is the force constant of the motor. $K_{a}$ is the tuneable acceleration feedback gain and $H_{a}$ is an optional shaping filter on the acceleration signal with DC gain of 1 . The sensor noises $n 1$ and $n 2$ are injected as disturbances in the units of sensor voltage. The cutting force $d F$, current noise $d I$ and position reference $r$ are also inputs to the closed loop system. 


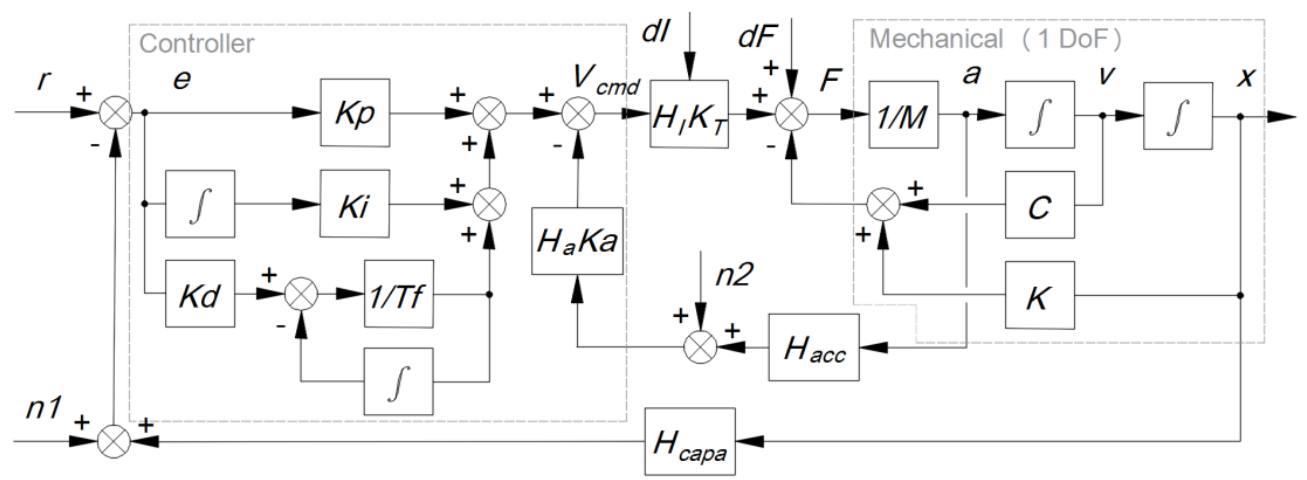

Figure 2: Analytical model of acceleration feedback control along with PID position controller

The frequency response of the two sensors can be approximated by second order low pass filters as:

$$
\begin{gathered}
H_{a c c}=\frac{V_{a c c}(s)}{X(s)}=\frac{\omega_{a c c}{ }^{2} S_{a c c}}{s^{2}+2 \omega_{a c c} s+\omega_{a c c}{ }^{2}} \\
H_{c a p a}=\frac{V_{c a p a}(s)}{X(s)}=\frac{\omega_{c a p a}{ }^{2} S_{c a p a}}{s^{2}+2 \omega_{c a p a} s+\omega_{c a p a}{ }^{2}}
\end{gathered}
$$

where $\omega_{\text {capa }}, \omega_{\text {acc }}$ are the measuring bandwidth $(-3 \mathrm{~dB})$ of the instruments and $S_{\text {capa }}, S_{a c c}$ are the sensitivity of the two sensors. $s$ is the complex frequency used in Laplace transform. The current loop transfer function from controller output voltage $V_{c m d}$ to motor current $I$ can also be modelled with a low pass characteristic with bandwidth $\omega_{I}$ and damping coefficient $\zeta$.

$$
H_{I}=\frac{I(s)}{V_{c m d}(s)}=\frac{\omega_{I}^{2}}{s^{2}+2 \zeta \omega_{I} s+\omega_{I}^{2}}
$$

According to the Newton's law, the dynamic behaviour of the moving body can be written as:

$$
\begin{gathered}
\frac{X(s)}{F(s)}=\frac{1}{M s^{2}+C s+K} \\
F(s)=H_{-} c t r(s)\left[r(s)-H_{\text {capa }} X(s)-n 1\right] H_{I} K_{T}-H_{a} K_{a}\left[H_{a c c} X(s) s^{2}+n 2\right] H_{I} K_{T}+d F(s)
\end{gathered}
$$

where $H_{-} c t r(s)$ is the transfer function of the PID controller, described as:

$$
H_{-} \operatorname{ctr}(s)=K_{p}+\frac{K_{i}}{s}+\frac{K_{d} s}{T_{f} s+1}
$$

where $K_{p}, K_{i}$ and $K_{d}$ are the PID gains respectively. $T_{f}$ is the time constant of the first order low-pass action on the derivative term (D). 


\subsection{Dynamic stiffness analysis}

The stiffness of a tool positioning system is evaluated by the amount of tool deviation caused by unit external force. It is a function of frequency because the stiffness value usually changes under excitations at different frequencies. Based on the analytical model in section 2.1, the dynamic compliance function, i.e. the ratio of cutting force disturbance $d F$ to tool position $x$, is the inverse of the dynamic stiffness. The dynamic compliance function can be derived as:

$$
\frac{X(s)}{d F(s)}=\frac{1}{\left(M+H_{a} K_{a} H_{a c c} H_{I} K_{T}\right) s^{2}+\left(C+\frac{K_{d}}{T_{f} s+1} H_{c a p a} H_{I} K_{T}\right) s+\left(K+K_{p} H_{c a p a} H_{I} K_{T}\right)+\left(K_{i} H_{c a p a} H_{I} K_{T}\right) \frac{1}{s}}
$$

Compared with Equation 4, the closed loop control system can be treated as a new mechanical setup. The disturbance force is the input and the tool position changes in response to the force. With the control action taking effects, the new setup shows quite different behaviours at different frequencies. The equivalent mass, spring and damping terms in the new setup are listed in Table 1.

Table 1: Equivalent terms of the closed loop system

\begin{tabular}{ccc}
\hline Name & Original & Equivalent in acceleration feedback control \\
\hline Mass term & $\mathrm{M}$ & $M+H_{a} K_{a} H_{\text {acc }} H_{I} K_{T}$ \\
Damping term & $\mathrm{C}$ & $\mathrm{C}+\frac{K_{d}}{T_{f} s+1} H_{\text {capa }} H_{I} K_{T}$ \\
Spring term & $\mathrm{K}$ & $K+K_{p} H_{\text {capa }} H_{I} K_{T}$ \\
Super spring term & -- & $K_{i} H_{\text {capa }} H_{I} K_{T}$ \\
\hline
\end{tabular}

The research by Schmidt et al. [27] shows that the spring term governs the low frequency response, the damping term controls the response near the resonant and the mass term determines the response beyond the closed loop bandwidth. Table 1 shows that the equivalent mass of the setup has been changed from $M$ to $M+H_{a} K_{a} H_{a c c} H_{I} K_{T}$ with acceleration feedback control. It is clear that no PID gains show up in the new mass term and this means the position control loop gains have no effect on the dynamic stiffness beyond the position closed loop bandwidth. There is a new super spring term 
introduced by the integral gain which doesn't exist in the original mechanical setup. This super spring effect is desired because it helps to achieve infinite DC stiffness. The damping and stiffness terms can also be changed by adjusting the $K_{d}, T_{f}$ and $K_{p}$ gains.

The reference tracking ability and disturbance rejection ability of a closed loop control system is mutually related. That means changing a single parameter will affect both the two transfer functions. This is not desired in the analysis because the motion control bandwidth is a benchmark in fast tool servo applications and should not be sacrificed in exchange for stiffness improvement. In order to compare the dynamic stiffness with different acceleration gains under the same reference tracking bandwidth, the reference tracking bandwidth is kept as unchanged as possible by adjusting the PID gains. The reference tracking function is derived as

$$
\frac{X(s)}{r(s)}=\frac{\left(K_{p}+\frac{K_{i}}{s}+\frac{K_{d} s}{T_{f} s+1}\right) H_{I} K_{T}}{\left(M+H_{a} K_{a} H_{a c c} H_{I} K_{T}\right) s^{2}+\left(C+\frac{K_{d}}{T_{f} s+1} H_{c a p a} H_{I} K_{T}\right) s+\left(K+K_{p} H_{\text {capa }} H_{I} K_{T}\right)+\left(K_{i} H_{c a p a} H_{I} K_{T}\right) \frac{1}{s}}
$$

The reference tracking transfer function and the disturbance rejection transfer function share the same denominators. In an ideal case when the current loop has a flat response characteristic and the acceleration sensor has an infinite measurement bandwidth with $H_{a}=1, H_{a c c}=S_{a c c}$, then the new mass term is a scaler. The numerator and denominator of Equation 8 can be multiplied by the global factor of $\mathrm{Kg}=M /\left(M+H_{a} K_{a} H_{a c c} H_{I} K_{T}\right)$ at the same time without changing the reference tracking function. In Lorentz type motion system, the original damping $C$ and stiffness $K$ are usually small compared to the control action $K_{d}$ and $K_{p}$. Thus this is equivalent to increasing the PID gains by the same ratio of $\mathrm{Kg}$. In the case when the new mass term is not a scaler but a function of the complex frequency $s$, the DC gain of this complex function will be used to calculate the increase factor.

Figure 3 a) shows an example of the analytical results for a positioning system with an ideal accelerometer with unlimited measuring bandwidth for acceleration feedback control. The dashed lines indicate the responses with PID control only and the solid lines show the responses with PID and acceleration feedback control. By introducing acceleration feedback and increasing the PID gains at the same time, the dynamic compliance function $X(s) / d F(s)$ is seen lower, which means an increased dynamic stiffness across all the frequency range. The reference tracking function $X(s) / r(s)$ 
and the closed loop phase margin are not changed at all. This means the acceleration feedback technique can theoretically improve the high frequency dynamic stiffness of a positioning system without the need for a larger moving mass.

\subsection{Influences of low-pass characteristics within the acceleration loop}

In practice, the acceleration measurement always has a low-pass feature limited by the bandwidth of the sensor and conditioning electronics. And the $H_{a}$ filter can be set by the user to maintain stability of the closed control loop. The filters acting on the acceleration signal will change the shape of the stiffness curve. When these low-pass characteristics exist in the acceleration loop, the transfer functions are plotted in Figure 3 b), with the same PID gains applied as in in Figure 3 a).

In the low frequency range, the dynamic stiffness is increased by the same level as in Figure 3 a). However, at frequencies just beyond the measurement bandwidth of the sensor, the dynamic stiffness deteriorates, showing larger amplitude. At even higher frequencies, the stiffness remains unchanged as the sensor cannot response to such high frequency motion any more.

Although the high frequency dynamic stiffness is not enhanced, the open loop transfer function shows larger phase margin compared with no acceleration feedback scenario, and therefore the system is more stable. With the larger phase margin available, the PID gains can be increased further because the bandwidth of the position loop is limited by the available phase margin. This helps to achieve both better low frequency disturbance rejection and larger reference tracking bandwidth.

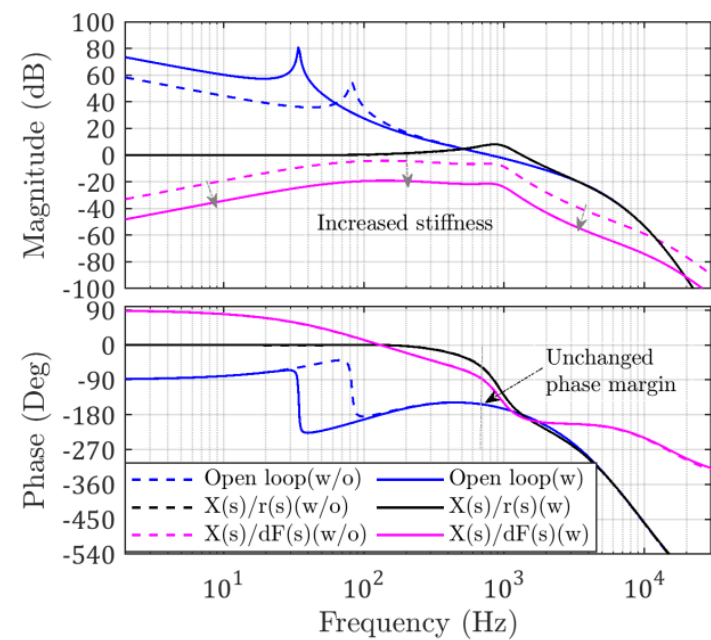

a) Acceleration feedback with an ideal sensor

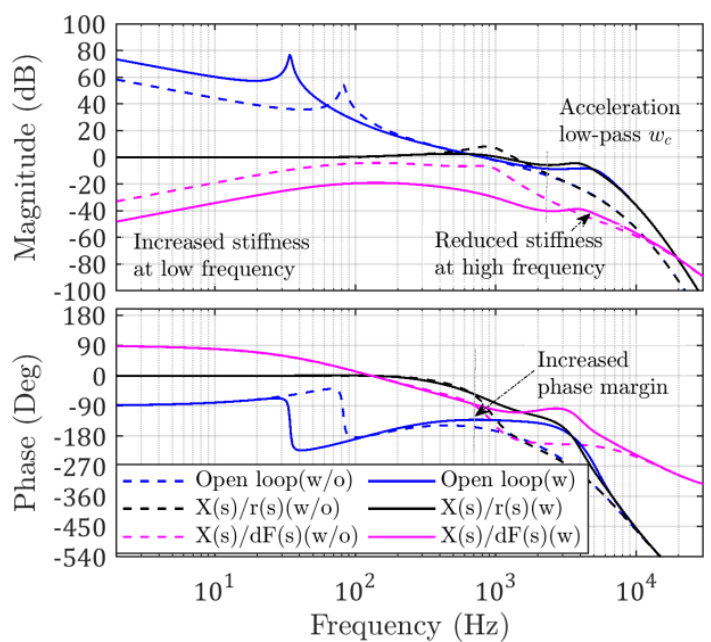

b) Acceleration feedback with low-pass characteristics

Figure 3: Comparison of loop transfer functions with (w) and without (w/o) acceleration feedback 


\subsection{Error contributions from the added acceleration sensor}

The additional acceleration control helps increasing the low frequency dynamic stiffness. However, the acceleration measuring sensor will inevitably bring some sensor noises which will contribute to the following errors of the cutting tool. In ultra-precision manufacturing scenario the tool positioning errors directly affects the surface quality of the finished parts [28,29]. Thus, it is necessary to study how much error will be introduced by the new sensor, providing a guideline for comparing the benefits of a higher stiffness and the side effects of the sensor noises.

The transfer functions from the two sensor voltage noises $n 1$ and $n 2$ to the closed loop tool position $X(s)$ can be described as:

$$
\begin{gathered}
\frac{X(s)}{n 1(s)}=\frac{X(s)}{r(s)} \\
\frac{X(s)}{n 2(s)}=\frac{X(s)}{d F(s)} H_{a} K_{a} H_{I} K_{T}
\end{gathered}
$$

It is found that the noise transfer function from the displacement sensor is the same as the closed loop reference tracking function. And the noise transfer function from the acceleration sensor is very similar to the dynamic compliance function, except the term of $H_{a} K_{a} H_{I} K_{T}$. In fact, the noise of the acceleration sensor voltage can be treated as a disturbance force after multiplied with $H_{a} K_{a} H_{I} K_{T}$. That is to say the acceleration feedback approach reduces the system response to the force disturbance but will introduce more disturbances at the same time.

A third disturbance source is the current noise $d I$ from the current loop controller. The currents in the motor winding will instantaneously generate force disturbances on the moving parts. So the current disturbances transfer function is

$$
\frac{X(s)}{d I(s)}=\frac{X(s)}{d F(s)} K_{T}
$$

The transfer functions of the three disturbance sources with different acceleration feedback depths are shown in Figure 4. The dashed lines are for a small acceleration gain (0.001) and the solid lines are for a larger acceleration gain (4). It can be seen that with a larger acceleration gain, the current noise disturbance is suppressed within the bandwidth of the acceleration sensor, showing similar shape with the dynamic compliance curve in Figure $3 \mathrm{~b}$ ). While the transfer function from the 
acceleration sensor noise shows significant increase with a larger acceleration gain. The noise from the capacitive sensor is redistributed from the middle frequency range to a higher frequency range.

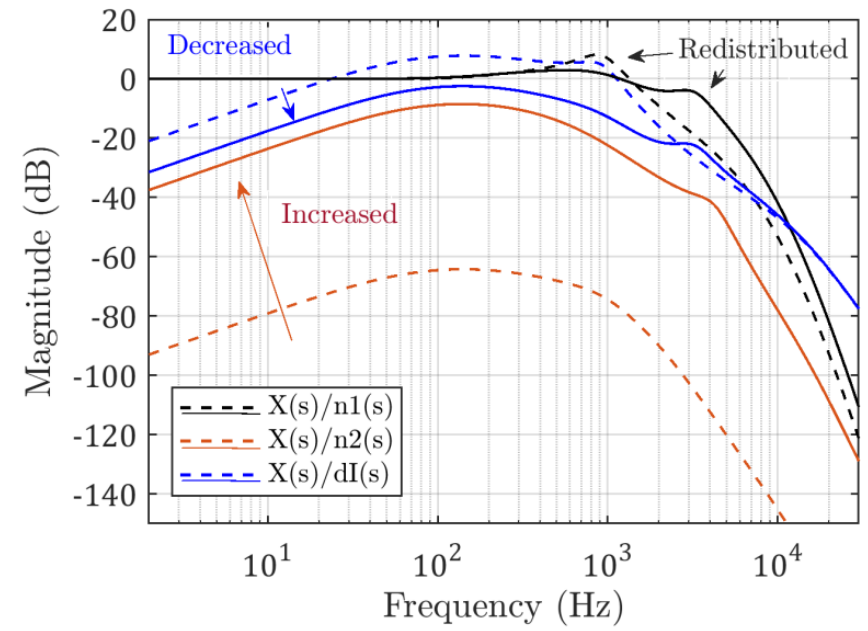

Figure 4: The transfer functions of the three disturbance sources with increasing acceleration feedback gains (Dashed: small acceleration gain; Solid: large acceleration gain)

Those error transfer functions together with pre-measured spectrums of the disturbances can be used to estimate the tool positioning error and following errors. The model of synthesising all the disturbance sources has been established in another paper [30]. And the same approach is used in the analysis of the new acceleration sensor error contribution in this paper. The results will be discussed later in Section 4.

\section{Experimental design}

A fast tool servo device shown in Figure 5 was specially designed for validation of the analytical model and analysis results. A custom-built flat Voice Coil Motor (VCM) was used to drive the tool and sensors assembly (shown in red). A diamond tool was fixed at the tip of the assembly and the whole assembly is free to move in the $\mathrm{Z}$ direction, guided by a pair of $\mathrm{E}$-shaped parallel flexure bearing. A capacitive displacement sensor and an accelerometer were fixed to the assembly. The dynamic characteristics of this setup were close to that of a single body mass-spring-damper system. 


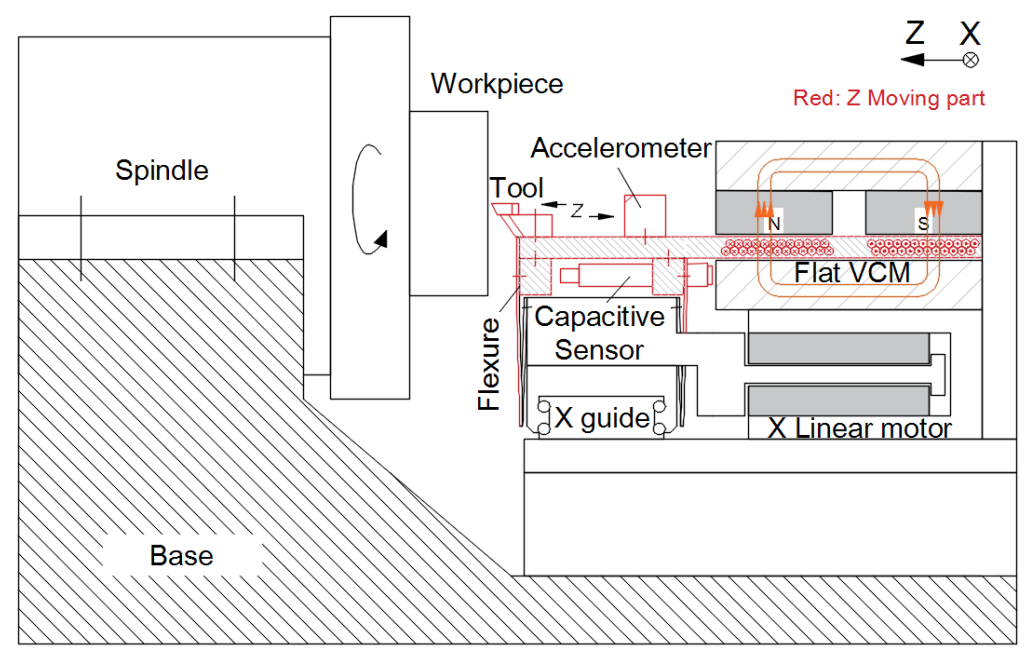

Figure 5: Fast tool servo system with accelerometers (the parts in red move in $\mathrm{Z}$ direction as a whole)

The built experiment device was mounted on a diamond turning machine bed as shown in Figure 6. A precision air bearing spindle was used to hold the workpiece for a facing cut. A copper sample was designed with a step change in height by milling half of the surface lower. Therefore, the cutting only occurred during half of the spindle revolution. The abruptly changed cutting force was used as the input excitation to evaluate the dynamic stiffness of the cutting system. The machining parameters for facing cut are listed in Table 2.

Table 2 Experimental conditions

\begin{tabular}{cc}
\hline Name & Value \\
\hline Workpiece material & Copper \\
Tool radius & $0.5 \mathrm{~mm}$ \\
Rake angle & 0 degree \\
Feed rate & $8 \mu \mathrm{m} / \mathrm{rev}$ \\
Depth of cut & $2 \mu \mathrm{m}$ \\
Spindle speed & $250 \mathrm{rpm}$ \\
Lubricant & Thin layer of mineral oil \\
\hline
\end{tabular}

The control algorithm was run on a custom-built Digital Signal Processor (DSP) board. The current loop was built by analogue circuits with a closed loop bandwidth of $400 \mathrm{kHz}(-3 \mathrm{~dB})$. A capacitive displacement sensor (Lion Precision CPL190) was used as the primary position feedback device and an accelerometer (PCB Electronics 333B50) were used to measure the acceleration of the cutting tool. The bandwidth of the acceleration sensor was set as $10 \mathrm{kHz}$. The control servo loop runs at $199.6 \mathrm{kHz}$ rate. Since the servo loop was updated at a high enough rate, the position and 
acceleration feedback action were calculated at the same DSP time slice. The machined surface was measured by a white light interferometer (ZYGO CP300).

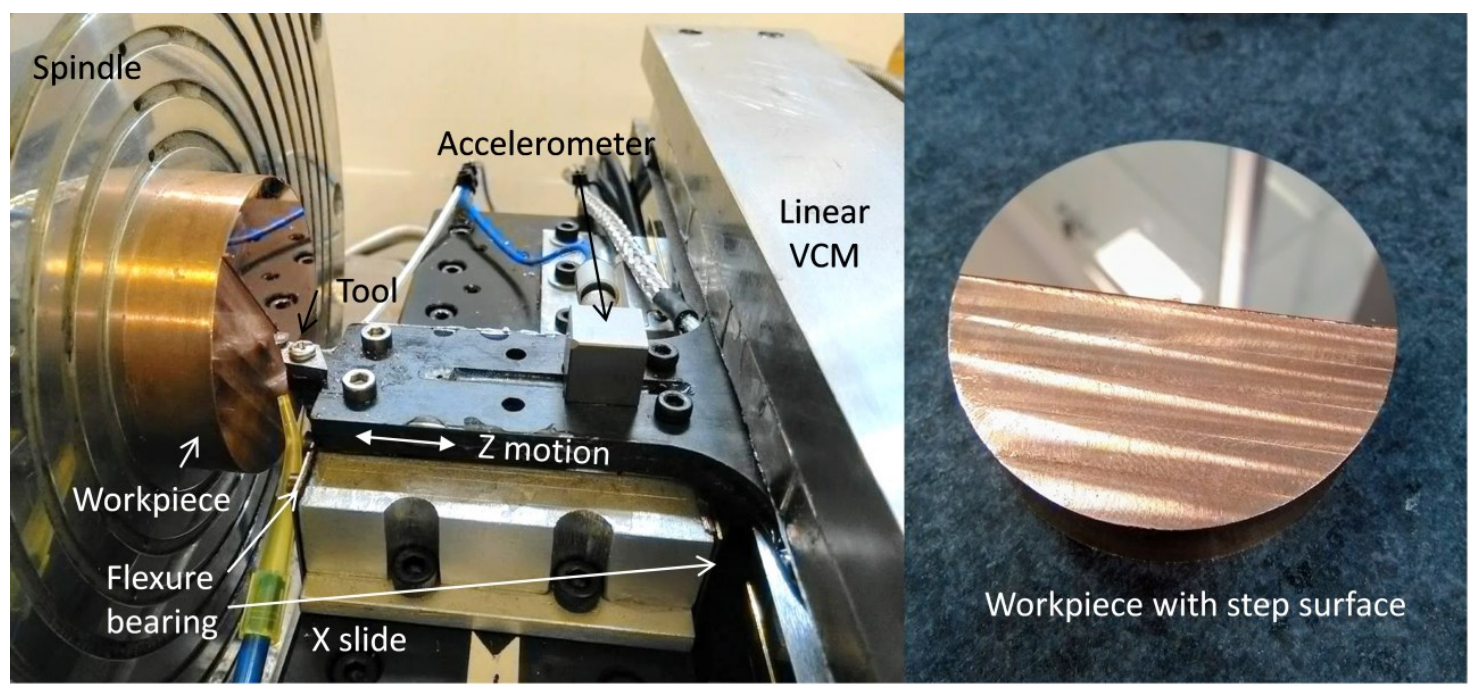

Figure 6: Experimental setup and the workpiece

\section{Results and discussions}

Firstly, the closed loop following errors with various acceleration gains are analysed and discussed. Then the dynamic stiffness is studied by sweep sinusoidal excitation tests. Lastly, the following errors under intermittent facing cut conditions are compared and the resulted surface profile is evaluated. The initial PID gains are selected as $K p=1.65, K i=562, K d=9.5 \times 10^{-4}$ and $T f=$ $9.6 \times 10^{-5}$

\subsection{Positioning errors introduced by the acceleration sensor}

The tool positioning following errors are acquired from the capacitive feedback sensor via the controller. The Power Spectrum Density (PSD) of the error signals under different acceleration gains are plotted in Figure 7. The position following error is mainly cause by sensor noises, the measuring error and the current noise as demonstrated in [30] for the same setup. In this paper, the additional error source from the acceleration sensor is added to the model. The power spectrum density of the acceleration sensor noise has been measured. The transmitted disturbance energies are calculated according to noise transfer functions and then summarised. If the acceleration feedback is disabled by setting the feedback gain $K a=0$, the error contribution from acceleration sensor will be zero according to Equation 10. When the acceleration feedback gain is set as 0.5 in Figure 7 a), the 
contribution of the acceleration sensor starts to show up with comparable level with the current noise. When the acceleration gain is increased further to $K a=4$ as in Figure $7 \mathrm{~b}$ ), the error contributed by the acceleration sensor noise surpasses the current noise and become the major error source. At the same time, because the reference tracking curve is raised near $5 \mathrm{kHz}$ frequency range, more noises from the capacitive sensor has been transmitted to the tool position within this frequency range. This is clearly shown from the change of the overall shape of the measured following error PSD in Figure $7 \mathrm{~b}$ ). The behaviours of the error contributions agree with the analysis results in Section 2.4.

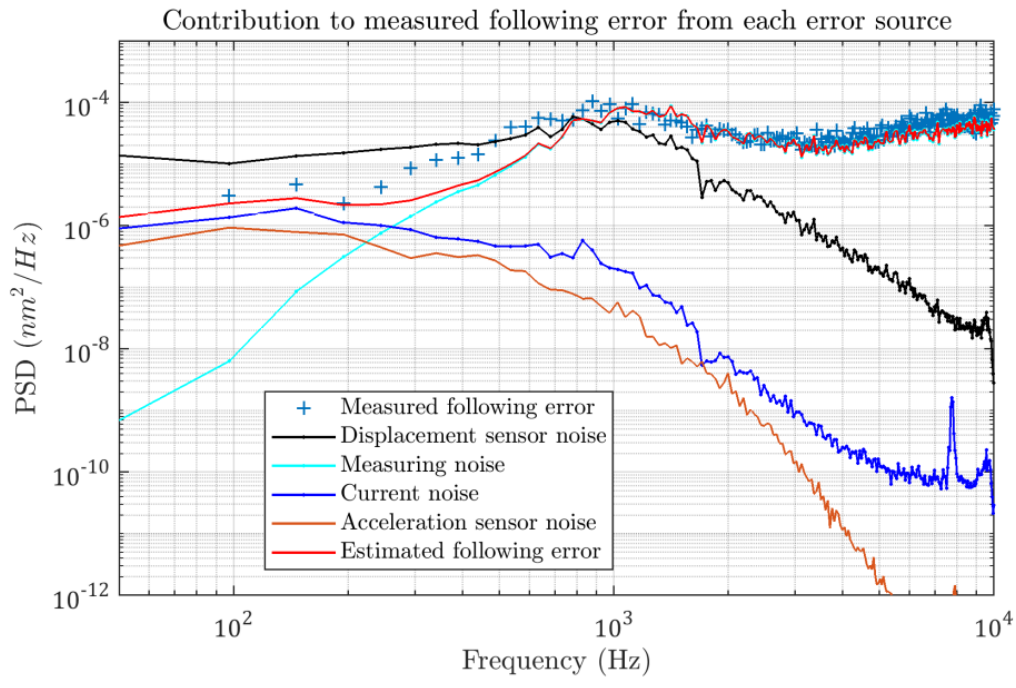

a) Measured following error with $\mathrm{Ka}=0.5$

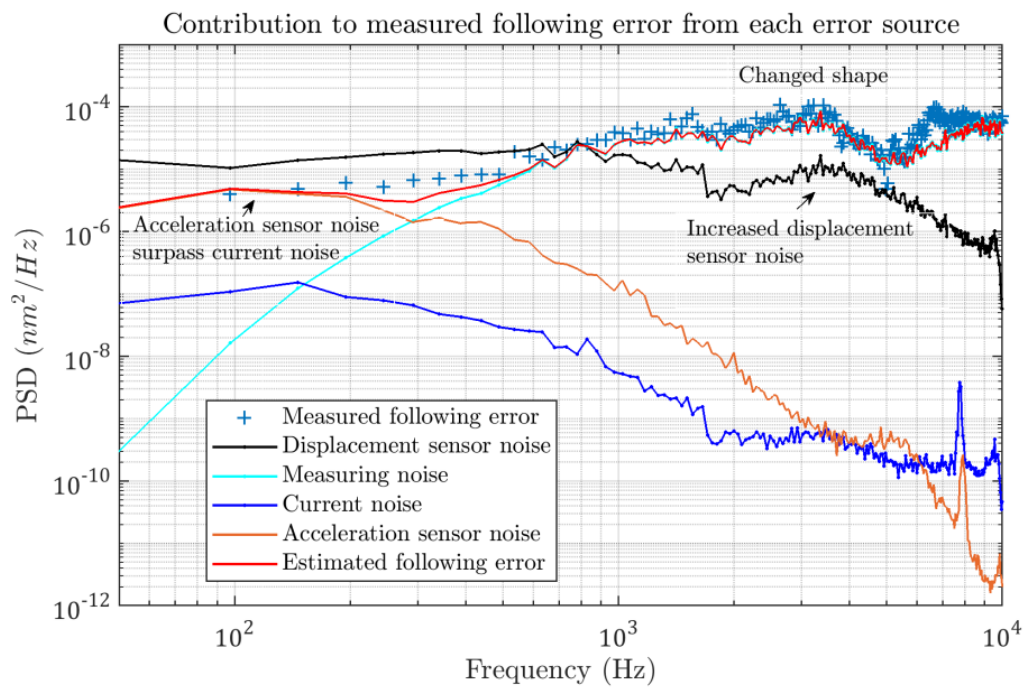

b) Measured following error with $\mathrm{Ka}=4$

Figure 7: Error contributions with different acceleration gains 


\subsection{Dynamic stiffness measurement}

The dynamic stiffness is tested by injecting sweep sinusoidal voltages onto the control output when the loop is closed. These disturbance voltages are considered proportional to disturbance forces since the current loop bandwidth is as high as $400 \mathrm{kHz}$ and the interested frequency range is only 10 $\mathrm{kHz}$. Figure 8 shows the measured frequency responses from disturbance force to position output with two different acceleration gains $(K a=0$ and $K a=1)$ and fixed PIDF gains $(K p=1.65, K i=$ $562, K d=9.5 \times 10^{-4}$ and $\left.T f=9.6 \times 10^{-5}\right)$. The modelled responses are plotted in Figure 8 as well to compare with the measured data. It can be seen that the measured data agrees with the model prediction for both cases. When the acceleration gain is increased, the response to cutting force at around $1 \mathrm{kHz}$ is more damped and thus the dynamic stiffness at this point is enhanced.

The stiffness at the low frequency range keeps the same despite the higher acceleration gains. This is because the spring term and the super spring term in Equation 7 are only related to the PID gains, which are not increased in this case. The increased damping is equivalent to a larger phase margin and it permits larger PID gains without loss of closed loop stability.

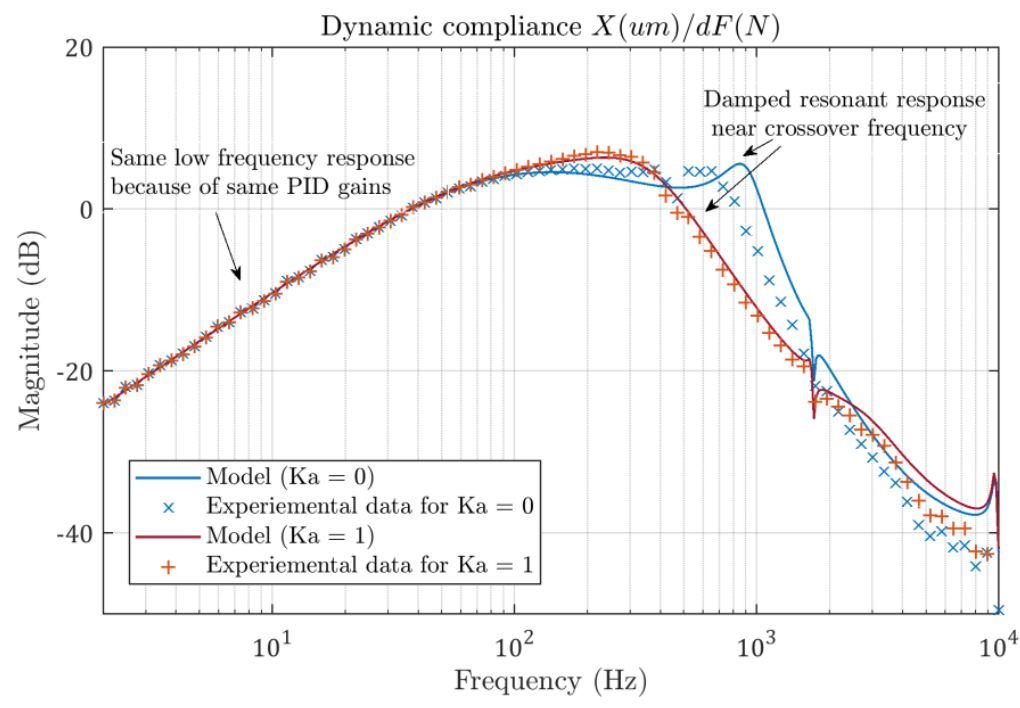

Figure 8: Dynamic compliance function with increasing $K a$ and fixed PID gains

When the PID gains are simultaneously increased by a global factor $\mathrm{Kg}$ as depicted in Section 2.2, the measured and modelled dynamic compliance curves are shown in Figure 9. It clearly shows that the measured stiffness at frequencies below $1 \mathrm{kHz}$ is increased by approximately 5.6 folds. And the measured response data agrees with the modelled one quite well. It is worth mentioning that the 
increased PID gains cannot be used without the acceleration gain because they will cause instability. Despite the improved low frequency stiffness, the stiffness at around $5 \mathrm{kHz}$ frequencies is worse than the no acceleration feedback scenario. This is due to the low-pass feature of the acceleration measurement, as discussed in Section 2.3.

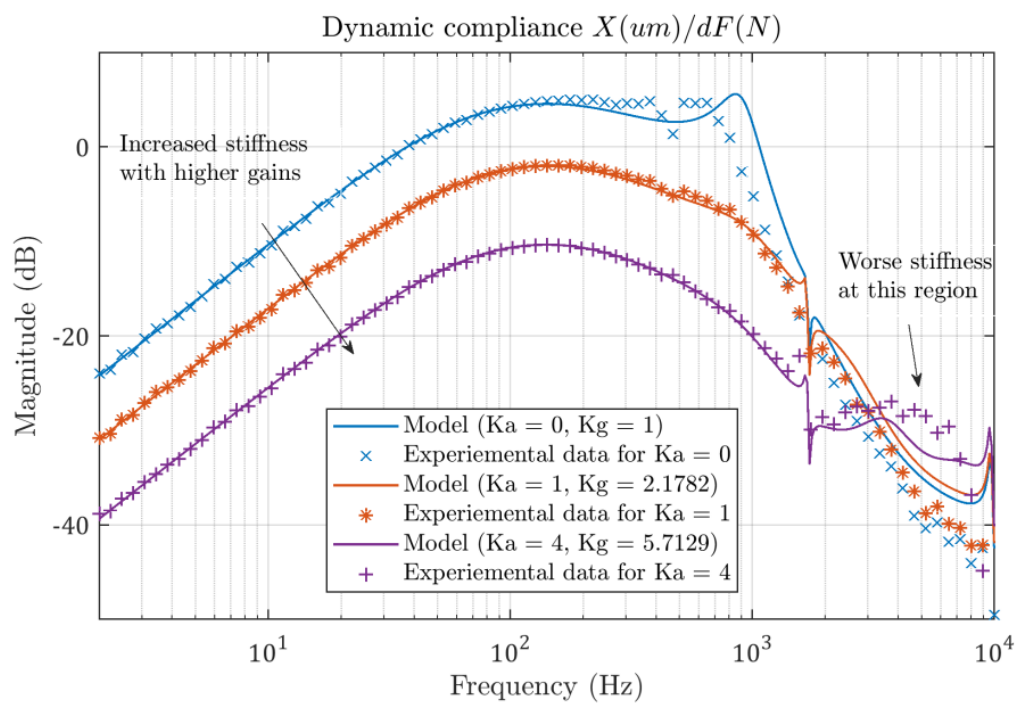

Figure 9: Dynamic compliance function with increasing $\mathrm{Ka}$ and proportionally increased PID gains

\subsection{Cutting experiment results}

The different parameters are deployed in the controller and used in the facing cut experiment. The acquired following error from the displacement sensor during the intermittent facing cut is shown in Figure 10. The acceleration gains are increased from 0 to 4 and the PID gains are increased accordingly in Figure 10 a) to d). Higher gains resulted in ringing noises and thus not shown here.

It can be seen from the first graph that when the tool enters the cutting region, there exists a sharp rise of the following error due to the sudden change of cutting force. Since the positive direction of the position sensor is defined as the direction away from the workpiece, this means less material will be cut. Then the tool was quickly controlled back to the commanded position. The duration is approximately 6 milliseconds for each peak. When the tool leaves the cutting region, there exists an opposite error peak due to the disappearance of the cutting force. The following error shows larger peak-to-valley values in the cutting region than the air cut region, which is reasonable because of the extra disturbance source. As the acceleration gains are increased, the value of the peak error decreases 
from $75 \mathrm{~nm}(K a=0)$ down to $25 \mathrm{~nm}(K a=4)$. The peak-to-valley errors during cutting are also seen lower with larger acceleration gains.

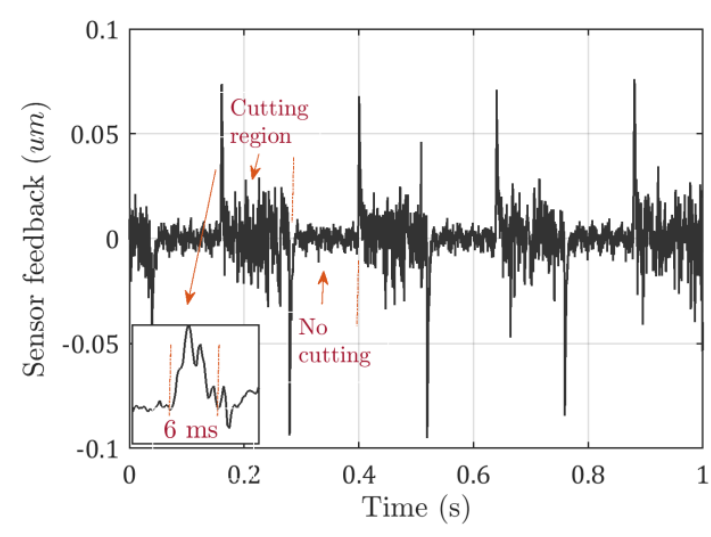

a) Position following error when $\mathrm{Ka}=0$

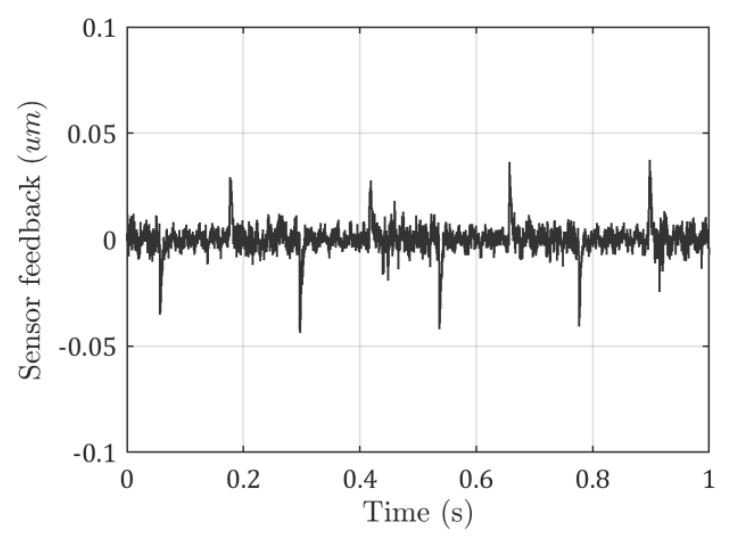

c) Position following error when $\mathrm{Ka}=1$

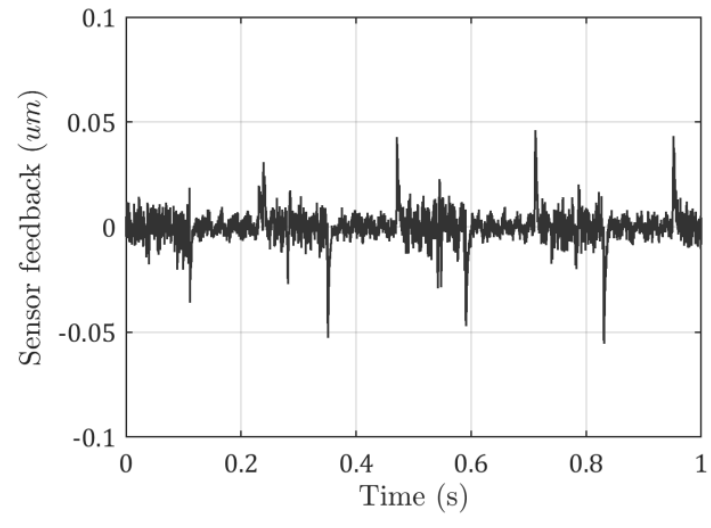

b) Position following error when $\mathrm{Ka}=0.5$

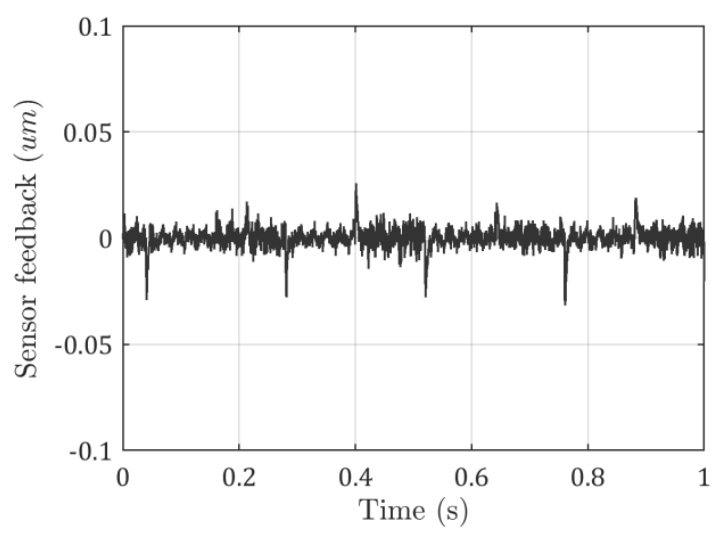

d) Position following error when $\mathrm{Ka}=4$

Figure 10: Following error caused by cutting force with different acceleration gains

Four circular tracks of $1 \mathrm{~mm}$ width are turned on the workpiece surface with the above four groups of gains. The measured surface topography is shown in Figure 11. The picture on the left is a stitched image which covers a surface area of $6.80 \mathrm{~mm} \times 5.15 \mathrm{~mm}$ and the pictures on the right show detailed surface form at the step edge of the first and last track measured with a $20 \mathrm{X}$ lens. Therefore, both low frequency and high frequency form errors can be captured. The cross sectional profiles along the cutting path (marked 4, 3, 2 and 1 in Figure 11 left) for the four cuts are shown in Figure 12. The profile is evaluated by averaging the cross sections within $0.2 \mathrm{~mm}$ width to the track centre in order to average out the random factor. The four tracks are found of slightly different height offset, which is considered as results of the thermal drifts between the cuts. The radius of the four tracks are $16.5 \mathrm{~mm}$, 
$17.5 \mathrm{~mm}, 18.5 \mathrm{~mm}$ and $19.5 \mathrm{~mm}$, corresponding to surface speed of $431 \mathrm{~mm} / \mathrm{s}, 458 \mathrm{~mm} / \mathrm{s}, 484 \mathrm{~mm} / \mathrm{s}$ and $510 \mathrm{~mm} / \mathrm{s}$, respectively.

The cross sectional profiles \#4 in Figure 12 shows obvious higher surface form close to the edge. This is attributed to the tool deviation caused by intermittent cutting force. As calculated from the 6 ms peak following error in Figure 10 and the surface speed of the workpiece, the surface form peak should be $2.586 \mathrm{~mm}$ in width. This agrees with the measured profile shape. As the acceleration gains are increased, the height of this form error is reduced from $106 \mathrm{~nm}(K a=0), 68 \mathrm{~nm}(K a=0.5)$, and $42 \mathrm{~nm}(K a=1)$ down to $27 \mathrm{~nm}(K a=4)$. This demonstrates that the increased gains help to achieve better stiffness at the low frequency range.

The detailed form errors for the track \#4 and track \#1 on the right in Figure 11 show obvious tool vibrations. The $0.05 \mathrm{~mm}$ and $0.056 \mathrm{~mm}$ error wavelength correspond to $8.62 \mathrm{kHz}$ and $9.1 \mathrm{kHz}$ tool vibrations respectively. It can be seen from these two graphs (with same colour bar setting) that the form is worse with a higher acceleration gain at the high frequency range. This result agrees with the measured stiffness curve in Figure 9. This experiment reveals that higher controller gains do not guarantee a better surface finish. Improvement of stiffness in some frequency ranges by increasing control gains also means deteriorated stiffness in other frequency ranges due to the nature of closed loop control. A properly tuned system is such that the stiffness is tuned higher where disturbance force is dominant, and the stiffness is allowed to be lower where the disturbance is negligible. The added acceleration sensor didn't break the law but expanded the tuneable frequency range to further than the position loop bandwidth. The step-changed force disturbance in this experiment is an extreme example since in some applications the cutting force might be increased smoothly by changing the depth of cut gradually. If the cutting force can be constrained within a low frequency range by proper programming, the acceleration feedback approach will be beneficial in improving machining quality. 

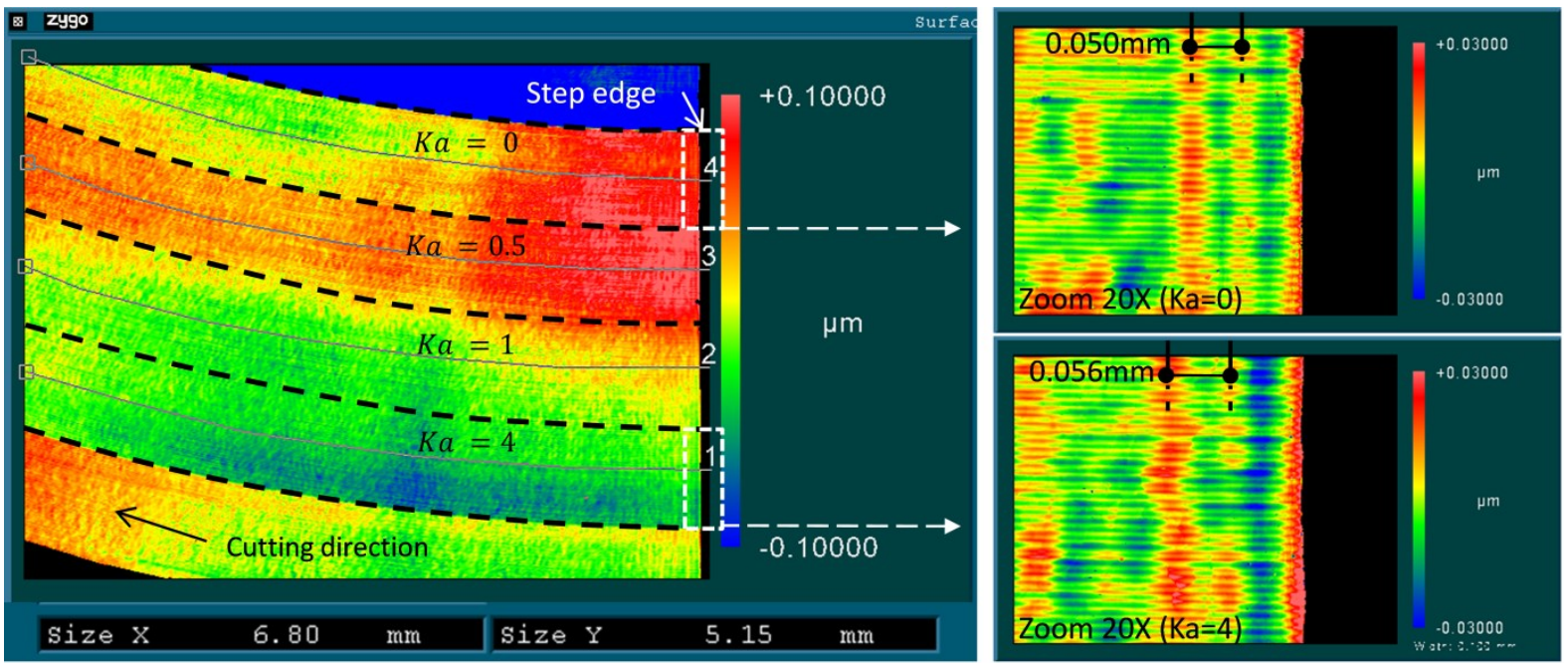

Figure 11: Measured surface topography with different acceleration gains and the detailed form error at the edge
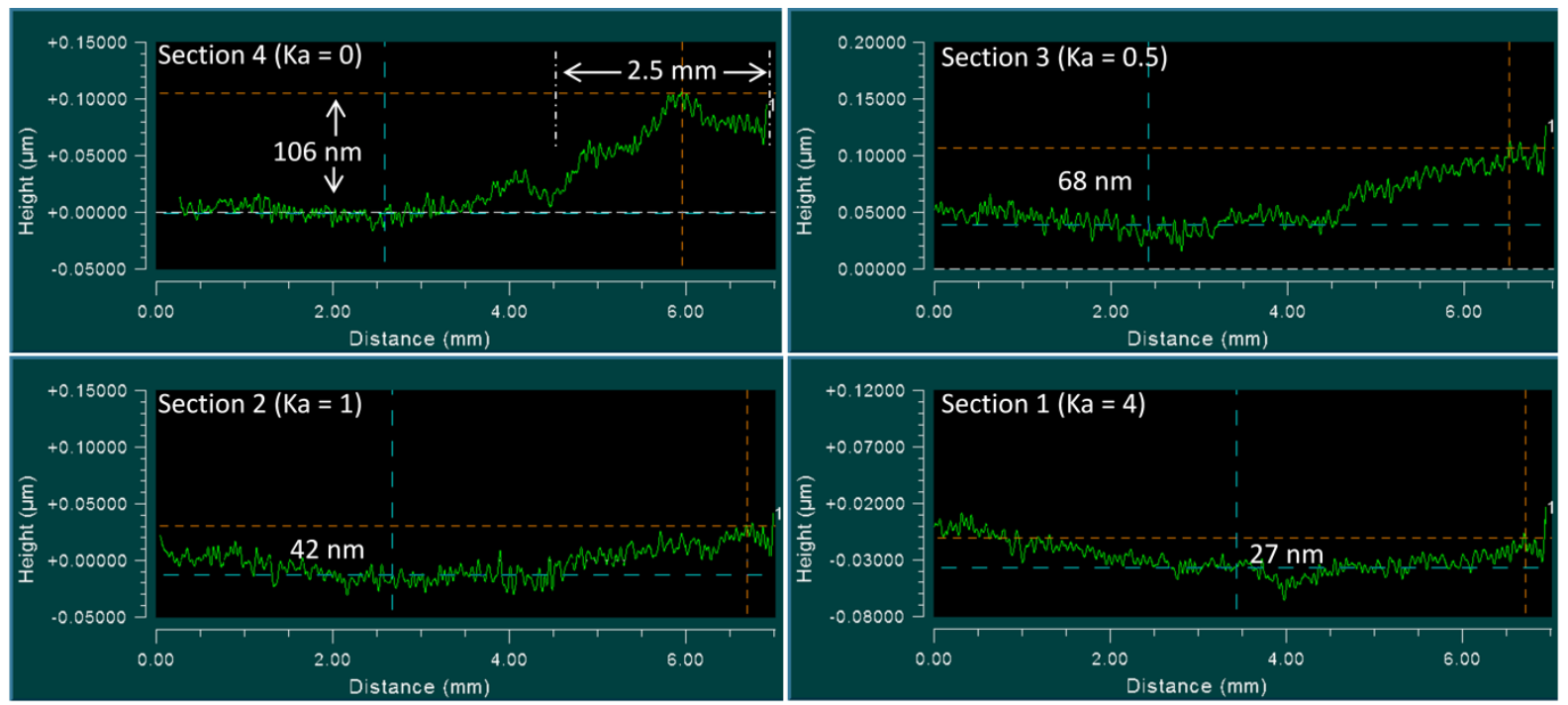

Figure 12: The averaged cross sectional profile along the cutting paths

\section{Conclusions}

In this paper, the effects of acceleration feedback control on performance of fast tool servo systems are studied considering the sensor imperfections. Theoretical models of the dynamic stiffness and error propagations are established and verified in the positioning and cutting experiments. The conclusions of this paper can be drawn as below:

a) The dynamic stiffness within the acceleration sensor bandwidth is improved for the positioning system. However, the stiffness is worse at frequencies just beyond the acceleration sensor bandwidth due to the existence of low-pass characteristics in the acceleration feedback loop. The acceleration 
feedback technique doesn't suppress the force disturbance at all frequencies but expands the frequency range where the stiffness curve can be tuned, according to the spectrum of disturbance.

b) The additional acceleration sensor brings new error source to the closed loop and its contribution increases with a larger acceleration gain. At the same time, the other disturbances are suppressed except the displacement sensor noise. There is a point where the increased acceleration error contribution surpasses others and starts to dominate, which is the upper limit for the practical acceleration gains in precision cutting.

\section{Acknowledgement}

The authors gratefully acknowledge the financial support from the UK Engineering and Physical Sciences Research Council (EPSRC) [EP/K018345/1]; and a joint John Anderson PhD studentship provided by DePuy (Ireland) and the University of Strathclyde [S150852] for this study. The first author also wants to thank China Scholarship Council for providing a stipend.

\section{Data Statement}

All data underpinning this publication are openly available from the University of Strathclyde Knowledge Base.

\section{Reference}

[1] S. Zhang, Y. Zhou, H. Zhang, Z. Xiong, S. To, Advances in ultra-precision machining of micro-structured functional surfaces and their typical applications, Int. J. Mach. Tools Manuf. 142 (2019) 16-41. doi:10.1016/J.IJMACHTOOLS.2019.04.009.

[2] H. Ma, J. Tian, D. Hu, Development of a fast tool servo in noncircular turning and its control, Mech. Syst. Signal Process. 41 (2013) 705-713. doi:10.1016/J.YMSSP.2013.08.011.

[3] H. Wang, S. Yang, Design and control of a fast tool servo used in noncircular piston turning process, Mech. Syst. Signal Process. 36 (2013) 87-94. doi:10.1016/J.YMSSP.2011.07.013.

[4] Z. Zhu, Z. Tong, S. To, X. Jiang, Tuned diamond turning of micro-structured surfaces on brittle materials for the improvement of machining efficiency, CIRP Ann. (2019). doi:10.1016/J.CIRP.2019.04.092.

[5] X. Liu, X. Zhang, F. Fang, Z. Zeng, H. Gao, X. Hu, Influence of machining errors on form errors of microlens arrays in ultra-precision turning, Int. J. Mach. Tools Manuf. 96 (2015) 8093. doi:10.1016/J.IJMACHTOOLS.2015.05.008.

[6] C.F. Cheung, S. To, T.C. Kwok, W.B. Lee, L.B. Kong, Analysis of surface generation in ultraprecision machining with a fast tool servo, Proc. Inst. Mech. Eng. Part B J. Eng. Manuf. 224 
(2010) 1351-1367. doi:10.1243/09544054jem1843.

[7] R. Neugebauer, B. Denkena, K. Wegener, Mechatronic Systems for Machine Tools, CIRP Ann. - Manuf. Technol. 56 (2007) 657-686. doi:10.1016/j.cirp.2007.10.007.

[8] F.R. Shaw, K. Srinivasan, Bandwidth enhancement of position measurements using measured acceleration, Mech. Syst. Signal Process. 4 (1990) 23-38. doi:10.1016/0888-3270(90)90038M.

[9] Y. Hori, Disturbance suppression on an acceleration control type DC servo system, in: 19th Annu. IEEE Power Electron. Spec. Conf., IEEE, 1988: pp. 222-229. doi:10.1109/PESC.1988.18137.

[10] R.D. Lorenz, Design Principles and Implementation of Acceleration Feedback to Improve Performance of dc Drives, IEEE Trans. Ind. Appl. 28 (1992) 594-599. doi:10.1109/28.137444.

[11] G. Ellis, R.D. Lorenz, Comparison of motion control loops for industrial applications, in: Conf. Rec. 1999 IEEE Ind. Appl. Conf. Thirty-Forth IAS Annu. Meet. (Cat. No.99CH36370), 1999: pp. 2599-2605. doi:10.1109/IAS.1999.799205.

[12] R.J.E. Merry, M.J.G. van de Molengraft, M. Steinbuch, Velocity and acceleration estimation for optical incremental encoders, Mechatronics. $20 \quad$ (2010) 20-26. doi:10.1016/J.MECHATRONICS.2009.06.010.

[13] H. Shim, M. Kochem, M. Tomizuka, Use of accelerometer for precision motion control of linear motor driven positioning system, in: Proc. 24th Annu. Conf. IEEE Ind. Electron. Soc., IEEE, 1998: pp. 2409-2414. doi:10.1109/IECON.1998.724102.

[14] J. Wittmann, R. Hagl, R. Kennel, Hybrid current and acceleration control of servo drives, Int. Exhib. Conf. Power Electron. Intell. Motion, Renew. Energy Energy Manag. (2017) 16-18. doi:10.1109/SBMicro.2017.7990714.

[15] F. Tian, K. Craig, M. Nagurka, Disturbance attenuation in a magnetic levitation system with acceleration feedback, Proc. IEEE Int. Conf. Ind. Technol. (2011) 59-64. doi:10.1109/ICIT.2011.5754346.

[16] M. Griese, J. Maas, Acceleration estimation and feedback for two-mass servo systems, IEEE/ASME Int. Conf. Adv. Intell. Mechatronics, AIM. (2017) 370-375. doi:10.1109/AIM.2017.8014045.

[17] A. Dumanli, B. Sencer, Optimal high-bandwidth control of ball-screw drives with acceleration and jerk feedback, Precis. Eng. 54 (2018) 254-268. doi:10.1016/J.PRECISIONENG.2018.06.002.

[18] S. Katsura, K. Irie, K. Ohishi, Wideband force control by position-acceleration integrated disturbance observer, IEEE Trans. Ind. Electron. 55 (2008) 1699-1706. doi:10.1109/TIE.2007.907664.

[19] H. Butler, Acceleration feedback in a lithographic tool, Control Eng. Pract. 20 (2012) 453464. doi:10.1016/j.conengprac.2011.12.008.

[20] A. Suzumura, Y. Fujimoto, T. Murakami, R. Oboe, A General Framework for Designing SISO-Based Motion Controller with Multiple Sensor Feedback, IEEE Trans. Ind. Electron. 63 (2016) 7607-7620. doi:10.1109/TIE.2016.2597133. 
[21] F. Chen, X. Lu, Y. Altintas, A novel magnetic actuator design for active damping of machining tools, Int. J. Mach. Tools Manuf. 85 (2014) 58-69. doi:10.1016/j.ijmachtools.2014.05.004.

[22] J. Munoa, X. Beudaert, K. Erkorkmaz, A. Iglesias, A. Barrios, M. Zatarain, Active suppression of structural chatter vibrations using machine drives and accelerometers, CIRP Ann. 64 (2015) 385-388. doi:10.1016/J.CIRP.2015.04.106.

[23] J. Abir, P. Morantz, S. Longo, P. Shore, A novel accelerometer based feedback concept for improving machine dynamic performance, in: Int. Fed. Autom. Control, Elsevier B.V., 2016: pp. 553-558. doi:10.1016/j.ifacol.2016.10.660.

[24] J. Abir, S. Longo, P. Morantz, P. Shore, Optimized estimator for real-time dynamic displacement measurement using accelerometers, Mechatronics. 39 (2016) 1-11. doi:10.1016/j.mechatronics.2016.07.003.

[25] M.A. Beijen, D. Tjepkema, J. van Dijk, Two-sensor control in active vibration isolation using hard mounts, Control Eng. Pract. 26 (2014) 82-90. doi:10.1016/J.CONENGPRAC.2013.12.015.

[26] D. Tjepkema, J. van Dijk, H.M.J.R.M.J.R. Soemers, Sensor fusion for active vibration isolation in precision equipment, J. Sound Vib. 331 (2012) 735-749. doi:10.1016/j.jsv.2011.09.022.

[27] R. Schmidt, G. Schitter, A. Rankers, The Design of High Performance Mechatronics-: HighTech Functionality by Multidisciplinary System Integration, IOS Press, 2014.

[28] S. Zhang, S. To, G.X. Zhang, Z. Zhu, A review of machine-tool vibration and its influence upon surface generation in ultra-precision machining, Int. J. Mach. Tools Manuf. 91 (2015) 34-42. doi:10.1016/J.IJMACHTOOLS.2015.01.005.

[29] S. Zhang, S. To, S.J. Wang, Z. Zhu, A review of surface roughness generation in ultraprecision machining, Int. J. Mach. Tools Manuf. 91 (2015) 76-95. doi:10.1016/j.ijmachtools.2015.02.001.

[30] F. Ding, X. Luo, W. Zhong, W. Chang, Design of a new fast tool positioning system and systematic study on its positioning stability, Int. J. Mach. Tools Manuf. 142 (2019) 54-65. doi:10.1016/J.IJMACHTOOLS.2019.04.008. 